\title{
Editorial—MTAP special issue on mobile media delivery
}

\author{
Christian Timmerer • Maria Martini • Jyrki Huusko
}

Published online: 8 February 2011

(C) Springer Science+Business Media, LLC 2011

The recent advances in wireless multimedia communications, including the provision of higher bit rates and more efficient compression methodologies, are enabling new services and applications and provide the technical means for multimedia communication systems becoming more portable, reliable, and ubiquitous. However, the delivery of multimedia content to every mobile handset on every mobile network, anytime and anywhere is still a major challenge.

Due to the interdisciplinary nature of mobile media delivery, several research projects from the mobile and wireless area, from the networked media area, as well as from the content technologies area, not neglecting security and experimental facilities, are dealing with mobile media delivery. The papers in the special issue were carefully selected among the submitted papers to illustrate the different aspects and different protocol levels of the mobile multimedia delivery. Our primary goal for this issue was to provide a holistic overview of multimedia delivery, taking into consideration the solutions from video coding, network and mobility management and radio techniques up to the evaluation of user experience.

For this mobile media delivery special issue, the authors were invited to submit contributions dealing with topics on mobile media delivery, including architectures for mobile media delivery, mobile media delivery (over next generation systems), peer-to-peer networks for mobile media delivery, solutions for optimized media delivery and transport over heterogeneous networks, quality of service/experience issues and solutions, including video quality assessment methods, network convergence for media delivery, media adaptation to networks and devices, cross-layer communication for mobile media delivery. At the source encoding side, works on (scalable) source coding for mobile media, joint optimisation of source and channel coding were invited. Parts of the articles were invited

C. Timmerer $(\bowtie)$

Department of Information Technology (ITEC), Multimedia Communication Group (MMC),

Klagenfurt University, Klagenfurt am Wörthersee, Austria

e-mail: christian.timmerer@itec.uni-klu.ac.at

M. Martini

Faculty of Computing, Information Systems and Mathematics,

Kingston University London, Kingston upon Thames, UK

J. Huusko

VTT Technical Research Centre, Oulu, Finland 
from a selection of the works presented at the $E U M O B$ workshop held in London in September 2009 and are representative of the research performed in the area within the framework of collaborative projects funded by the European Commission.

Assessing the end-to-end quality perceived by the user is a critical step in the design of mobile media delivery systems. The article "A hybrid method for the context of use: evaluation of user-centered quality of experience for mobile (3D) television" reports the results of extensive psychoperceptual quality evaluation experiments used to assess the resulting audiovisual quality from fundamental signal processing algorithms to consumer services. The paper presents a method for evaluating the experienced multimedia quality in the context of use. Three studies are presented, where the quality of mobile 2D and 3D television is assessed in three different field contexts, one simulated context and one controlled laboratory situation when audio-video compression and transmission parameters were varied.

In order to achieve satisfactory quality of experience for the user it has been demonstrated that cross-layer design, i.e., the joint design of the different layers of the protocol stack, is in general beneficial for mobile media delivery.

A group of articles address the actual transmission based on unequal loss/error protection of video streams and cross-layer design aspects. The article "Priority-based Media Delivery using SVC with RTP and HTTP Streaming" proposes a media delivery methodology for scalable video coding (SVC) to overcome link interruptions and channel bitrate reduction in mobile networks, by performing a transmission scheduling where media data are treated unequally depending on their importance. The presented approach comprises a media priority-based pre-buffer to overcome phases under reduced connectivity. In order to overcome interruptions and intervals with reduced bitrate, the methodology is used to achieve longer continuous playback than the traditional approach without stops in the video playout and, thus, improves the video playback quality. The methodology is analysed in the traditional RTP streaming and in the adaptive HTTP streaming context. The benefits of using $\mathrm{SVC}$ is presented in terms of received quality during interruption and re-buffering time. The article "Image and video transmission: a comparison study of using unequal loss protection and multiple description coding" compares multiple description coding (MDC) and unequal loss protection (ULP) as tools to deliver multimedia data to mobile users. Graceful degradation encoding schemes are able to cope with packet losses due to timevariant channels as they allow the receivers to partially reconstruct the transmitted data with only a subset of received packets. Rate-distortion-based multiple description coding and a state-of-the-art unequal loss protection algorithm based on Reed Solomon Forward Error Correction (FEC) codes are addressed. The comparison is performed using as a case study JPEG 2000 coded images and advanced video coding (AVC) sequences transmitted over lossy packet networks. Complexity aspects are also considered. The article "Optimisation of Multimedia over wireless IP links via X-layer design: an end-to-end transmission chain" presents the architecture set-up proposed within the ICT FP7 OPTIMIX project to study innovative solutions enabling enhanced multimedia streaming in a point to multi-point context for an IP based wireless heterogeneous system, based on cross layer adaptation of the whole transmission chain. The corresponding simulation chain architecture is detailed with the description of the existing and/or future features of each module.

Finally, multiple papers deal with various architectural aspects related to mobile media delivery. The article "Distributed information service architecture for overlapping multiaccess networks" presents and evaluates a distributed information service which can enhance (mobile) media delivery, e.g., over multiple access networks such as 3G/HSPA, 
WLAN, and WiMAX. The information service introduces a new distributed control and management framework (DCMF) and a mobility management triggering functionality (TRG) which is evaluated on a test-bed comprising the aforementioned access networks. The article "Annotation based personalized adaptation and presentation of videos for mobile applications" describes an architecture for personalized video adaptation and presentation for mobile applications which is guided by automatically generated annotations. These annotations provide means for more intelligent adaptation techniques which primarily reduce the quality of unimportant regions in case a bit rate reduction becomes necessary. Additionally, a presentation layer is added to enable advanced multimedia viewers to adequately present the interesting parts of a video in case the user wants to zoom in. Finally, the article "Adaptive IPTV Services based on a novel IP Multimedia Subsystem" introduces new functionalities in IPTV over 3GPPs' IP Multimedia Subsystem (IMS) architecture which aims to optimize both the satisfaction of the end-user and resource utilization of the operators' network(s). Therefore, a contextsensitive user profile model is used to deliver IPTV streams adapted to the specifics of the users' environment taking into account the impact of spatio-temporal dynamics of the video content on the deduced perceptual quality in order to optimize the operators' network utilization. In this context, the article introduces a Multimedia Content Management System (MCMS) to perform dynamic cross-layer adaptation of the IPTV stream based on perceived QoS measurements at the end-user side.

We thank all authors for their contributions to this special issue. In particular, the editors would like to thank the reviewers for their hard work who significantly helped to improve the initial submissions.

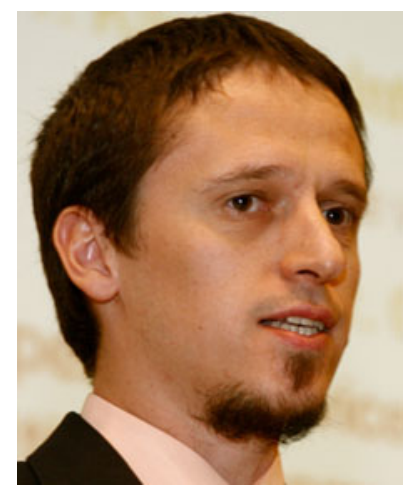

Christian Timmerer is an assistant professor at the Department of Information Technology (ITEC), Multimedia Communication Group (MMC), Klagenfurt University, Austria. His research interests include the transport of multimedia content, multimedia adaptation in constrained and streaming environments, distributed multimedia adaptation, and Quality of Service/Quality of Experience. He was the general chair of WIAMIS 2008 and EUMOB 2009 and has participated in several EC-funded projects, notably DANAE, ENTHRONE, P2P-Next, and ALICANTE. He is an Associate Editor for IEEE Computer Science Computing Now, Area Editor for Elsevier Signal Processing: Image Communication, and a Key Member of the Interest Group on Image and Video Coding of IEEE MMTC. He also participated in ISO/MPEG work for several years, notably in the area of MPEG-21, MPEG-M, MPEG-V, and DASH. He received his PhD in 2006 from the Klagenfurt University. Publications and MPEG contributions can be found under http://research.timmerer.com, follow him on http://www.twitter.com/timse7, and subscribe to his blog http://blog.timmerer.com. Full bio can be found at http://www-itec.uni-klu.ac.at/ timse/cv/. 


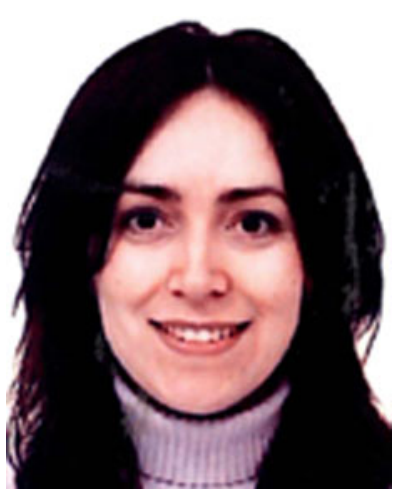

Maria Martini is a Senior Lecturer in the Faculty of Computing, Information Systems and Mathematics in Kingston University, London, where she is also coordinating the Wireless Multimedia Networking Research Group. She received the Laurea in electronic engineering (summa cum laude) from the University of Perugia (Italy) in 1998 and the Ph.D. in Electronics and Computer Science from the University of Bologna (Italy) in 2002. She has worked as a key person for national and international projects, such as the JSCC project, with Philips Research, the JOCO and PHOENIX European IST projects. She is currently leading the KU team in the ICT-OPTIMIX European project and she is principal investigator in a number of national and industry-funded research projects. An IEEE Senior Member, she is a (guest) editor and reviewer for international journals and she is/was in the organising and programme committee of several international conferences. She was general chair of the ICST/ACM MOBIMEDIA 2009 conference and of EUMOB 2008. She is now organising the IEEE Workshop on Streaming and Media Communications (ICME 2011) and the First International Workshop on Cross-Layer Operation Aided Multimedia Streaming (IEEE VTC 2011). She coordinated (2008-2010) the edition of the Strategic Applications Agenda (SAA) on mobile health and inclusion applications in the eMobility European Technology Platform. She is part of international committees and expert groups, including the IEEE Multimedia Communications technical committee (key member 2010-2012 for QoE and multimedia streaming). Her research interests include wireless multimedia networks, cross-layer design, joint source and channel coding, 2D/3D error resilient video, 2D/3D video quality assessment, and medical applications. She is the author of over 50 scientific articles and book chapters, and the inventor of several patents on wireless video.

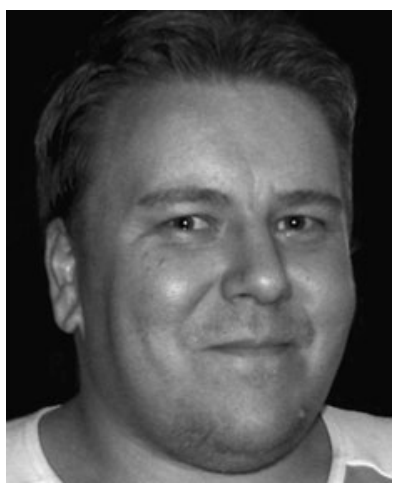

Jyrki Huusko is a research team leader at VTT Technical Research Centre in Network Technologies' Seamless Networking team. His team main focus is on wireless Internet research, novel content distribution technologies and cognitive network solutions for multimedia delivery. His past research topics have also included lowly parallel computer clusters and distributed computing, IPv6 networking, cross-layer communications design in heterogeneous wireless and mobile networks and cross-layer communication aided network mobility, multi-access and multimedia delivery. His current research topics include especially future autonomic networks and services, future Internet architectures, content centric networking and 
cognitive network solutions for multimedia delivery optimisation. He has also acted as a reviewer in several international conferences and journals, conference and workshop organisation general chair (e.g. ICST MobiMedia 2008, EUMOB 2009), advisory board member of MobiMedia, and technical program committee member in variety of network technology and multimedia delivery conferences and journal quest editor. During his career at VTT, he has participated actively in variety of European Commission funded IST/ICT 5th, 6th and 7th Framework Programme projects such as UNIVERSELF, 4WARD, BIONETS, PHOENIX, WEIRD, WIND-FLEX as a researcher and management team member. 